\title{
Effects of synbiotic supplementation on insulin resistance in subjects with the metabolic syndrome: a randomised, double-blind, placebo-controlled pilot study
}

\author{
Tannaz Eslamparast ${ }^{1,2}$, Farhad Zamani ${ }^{3}$, Azita Hekmatdoost ${ }^{1 *}$, Maryam Sharafkhah ${ }^{2}$, Sareh Eghtesad $^{2}$, \\ Reza Malekzadeh ${ }^{2}$ and Hossein Poustchi ${ }^{2}$ * \\ ${ }^{1}$ Department of Clinical Nutrition and Diet Therapy, Faculty of Nutrition and Food Technology, National Nutrition \\ and Food Technology, Research Institute Shabid Beheshti University of Medical Science, National Nutrition and \\ Food Technology Research Institute, Shabid Beheshti University of Medical Science, Tebran, Iran \\ ${ }^{2}$ Liver and Pancreatobiliary Diseases Research Center, Digestive Diseases Research Institute, \\ Tehran University of Medical Sciences, Shariati Hospital, Tebran, Iran \\ ${ }^{3}$ Gastroenterology and Liver Disease Research Center, Firoozgar Hospital, Iran University of Medical Sciences, \\ Tebran, Iran
}

(Submitted 15 January 2014 - Final revision received 10 March 2014 - Accepted 26 March 2014 - First published online 22 May 2014)

\begin{abstract}
To evaluate the effects of synbiotic supplementation on insulin resistance and lipid profile in individuals with the metabolic syndrome, we conducted a randomised, double-blind, placebo-controlled pilot study on thirty-eight subjects with the metabolic syndrome; they were supplemented with either synbiotic capsules containing 200 million of seven strains of friendly bacteria plus fructo-oligosaccharide or placebo capsules twice a day for 28 weeks. Both the synbiotic (G1) and the placebo (G2) groups were advised to follow an energy-balanced diet and physical activity recommendations. Parameters related to the metabolic syndrome and insulin resistance were measured every 7 weeks during the course of the study. After 28 weeks of treatment, the levels of fasting blood sugar and insulin resistance improved significantly in the $\mathrm{G} 1$ group $(P<0 \cdot 001)$. Both the $\mathrm{G} 1$ and $\mathrm{G} 2$ groups exhibited significant reductions in TAG levels $(-71 \cdot 22 v$. $-10.47 \mathrm{mg} / \mathrm{dl}(-0.80 v . \quad-0.12 \mathrm{mmol} / \mathrm{l})$ respectively; $P<0.001)$ and total cholesterol levels $(-21.93 \quad v . \quad-14.2 \mathrm{mg} / \mathrm{dl} \quad(-0.57 \quad v$. $-0.37 \mathrm{mmol} / \mathrm{l})$ respectively; $P=0.01)$, as well as increases in HDL levels $(+7.7 v \cdot+0 \cdot 05 \mathrm{mg} / \mathrm{dl}(+0 \cdot 20 v \cdot+>0 \cdot 01 \mathrm{mmol} / \mathrm{l}) \mathrm{respectively}$; $P<0.001)$. The mean differences observed were greater in the G1 group. No significant changes were observed in LDL levels, waist circumference, BMI, metabolic equivalent of task and energy intake between the groups. The present results indicate that synbiotic supplementation increases the efficacy of diet therapy in the management of the metabolic syndrome and insulin resistance.
\end{abstract}

Key words: Metabolic syndrome: Synbiotic supplementation: Insulin resistance: Gut flora

The metabolic syndrome is a rapidly growing worldwide pandemic that increases the risk of developing many chronic diseases, especially CVD and type 2 diabetes $^{(1)}$. Although several aetiological factors are involved in the development of the metabolic syndrome, insulin resistance and obesity play a key role. Recently, gut microbiota has been considered to be a regulator of energy homeostasis and ectopic fat deposition due to its effects on metabolic disorders ${ }^{(2,3)}$. In particular, studies comparing obese $v$. matched lean individuals have found a shift in bacterial phyla and observed more Firmicutes and less Bacteroidetes in the distal gut of obese subjects, and this alteration was found to be abolished after diet-induced weight loss ${ }^{(4,5)}$. Pathogens in the gut can decrease the permeability of the intestinal wall and enter the circulation, where they induce metabolic and inflammatory cascades. Recent evidence has indicated that circulating lipopolysaccharides (LPS), components of Gram-negative bacterial cell walls, are correlated with insulin levels, glucose levels and insulin resistance (as measured by homeostasis model assessment - insulin resistance $($ HOMA-IR $))^{(6,7)}$. In addition, high

Abbreviations: DDRI, Digestive Diseases Research Institute; G1, synbiotic; G2, placebo; HOMA-IR, homeostasis model assessment - insulin resistance; LPS, lipopolysaccharides; MET, metabolic equivalent of task; NNFTRI, National Nutrition and Food Technology Research Institute; QUICKI, quantitative insulin sensitivity check index; TC, total cholesterol.

*Corresponding authors: A. Hekmatdoost, fax +98 2122360657, email a_hekmat2000@yahoo.com; H. Poustchi, fax +98 2182415400, email h.poustchi@gmail.com 
levels of TAG and low levels of HDL-cholesterol that often exist during insulin resistance and the metabolic syndrome are the most essential factors involved in LPS molecule conservation in the circulation ${ }^{(8)}$.

It has been suggested that probiotics and prebiotics might counteract the development of the metabolic syndrome by replacing the aggravating bacteria in the gut, which in turn can improve serum lipid levels and insulin resistance ${ }^{(9)}$. Currently, it has been shown in preclinical studies that probiotics are effective at attenuating the metabolic syndrome; however, only few pilot studies in human subjects have been carried out with promising preliminary results ${ }^{(10-12)}$. Previous studies have reported that the synergistic effects of synbiotic supplementation on the intestinal and faecal microflora and immune system are significantly greater than the effects of either prebiotic or probiotic supplementation alone ${ }^{(13,14)}$. Considering the various beneficial effects of prebiotics and probiotics and the lack of published data on this issue, the present study was designed to evaluate the effects of supplementation with a type of synbiotic on insulin resistance and serum lipid levels in subjects with the metabolic syndrome.

\section{Research design and methods}

\section{Participants}

The present study was a prospective, randomised, doubleblind, placebo-controlled study. Men and women aged 18 years and above with a diagnosis of the metabolic syndrome were selected from the Haraz clinic of Amol. The metabolic syndrome was diagnosed as having three of the following five features: increased waist circumference $(\geq 102 \mathrm{~cm}$ in men and $\geq 88 \mathrm{~cm}$ in women); elevated TAG levels $(\geq 150 \mathrm{mg} / \mathrm{dl} \quad(\geq 1.69 \mathrm{mmol})) ; \quad$ reduced HDL-C levels $(<40 \mathrm{mg} / \mathrm{dl} \quad(<1.03 \mathrm{mmol} / \mathrm{l})$ in men and $<50 \mathrm{mg} / \mathrm{dl}$ $(<1.29 \mathrm{mmol} / \mathrm{l})$ in women); elevated blood pressure $(\geq 130 /$ $85 \mathrm{mmHg}$ or on treatment for hypertension); elevated glucose levels $(\geq 100 \mathrm{mg} / \mathrm{dl} \quad(\geq 5.55 \mathrm{mmol} / \mathrm{l}))$, according to the National Cholesterol Education Program Adult Treatment Panel III report ${ }^{(15)}$, and the diagnosis was confirmed via careful questioning by the primary physician and dietitians. Individuals with decompensated diabetes mellitus, untreated hypothyroidism, clinically or biochemically recognised systemic diseases, and psychiatric disorders impairing their ability to provide written informed consent, as well as pregnant/ breast-feeding women or those of child-bearing age without effective birth control use, were excluded from the study. If a participant was found to have missed $\geq 10 \%$ of his or her supplement dose at follow-up, he or she was also excluded from the study. The present study was registered at ClinicalTrials.gov ID: NCT02008838.

\section{Study design}

A total of 102 individuals were assessed for participation eligibility; of these, forty met the inclusion criteria of the study and two declined to participate, resulting in a sample size of thirtyeight participants (Fig. 1). The thirty-eight participants were then randomly assigned to the synbiotic (G1;n 19) group or the placebo (G2;n 19) group. The G1 group was given a synbiotic supplementation (as a capsule), while the G2 group was given an identical appearing placebo capsule (250 mg maltodextrin) twice a day for 28 weeks. The participants were instructed to consistently take the capsules $2 \mathrm{~h}$ after consumption of the same meal each day.

Each synbiotic capsule (Protexin) contained $2 \times 10^{8}$ colonyforming units of seven strains of friendly bacteria (Lactobacillus casei, Lactobacillus rhamnosus, Streptococcus thermophilus, Bifidobacterium breve, Lactobacillus acidophilus, Bifidobacterium longum and Lactobacillus bulgaricus), prebiotics (250 mg fructo-oligosaccharide) and a probiotic culture (magnesium stearate (source: mineral and vegetable) and vegetable capsule (hydroxypropyl methylcellulose)). As previous studies had used various combinations of VSL\#3, Lactobacillus longum and fructo-oligosaccharide, demonstrating their beneficial effects on intestinal microflora ${ }^{(16-18)}$, this synbiotic capsule was chosen for the present study as it contained all these strains in addition to others.

A 7-week supply of capsules was given to the participants at the time of randomisation and the remainder at the time of the 7th-, 14th- and 21st-week follow-up visits. The participants, investigators and staff were blinded to the treatment assignment until the end of the study. All the participants signed an informed consent form after a full review of the risks and benefits of the study, which were approved by the ethics committee of the National Nutrition and Food Technology Research Institute (NNFTRI) of Shahid Beheshti University of Medical Sciences and the Digestive Diseases Research Institute (DDRI) of Shariati Hospital, Tehran, Iran.

An interviewer completed a questionnaire for each participant, which contained information on each individual's past medical history, family history and medication history, as well as lifestyle behaviours such as smoking, drinking, diet and exercise. Both groups were counselled to follow an energy-balanced diet and physical activity recommendations based on the Clinical Guidelines on the Identification, Evaluation, and Treatment of Overweight and Obesity in Adults from the National Institutes of Health and the North American Association for the Study of Obesity ${ }^{(19,20)}$. Each individual was advised to follow a diet containing 2092-4184kJ less energy than his or her usual daily intake. The distribution of nutrients in relation to the total energy value was as follows: total fat $\leq 30 \%$ total energy value; SFA 8-10\%; MUFA 15\%; PUFA 5\%; protein 10-15\%; carbohydrates 50-60\%; dietary cholesterol $<300 \mathrm{mg} / \mathrm{d}$; fibre $20-30 \mathrm{~g} / \mathrm{d}$. They were also advised to perform at least $20-30 \mathrm{~min}$ of high-intensity exercise $3-4 \mathrm{~d}$ per week ${ }^{(21)}$.

\section{Follow-up}

The initial and follow-up visits took place at Haraz clinic. After baseline data collection, follow-up assessments were performed at weeks 7, 14 and 21 and during the last visit, at week 28. Phone calls were also made to each participant on a monthly basis to assess compliance with the study supplementation and the development of any adverse events. 


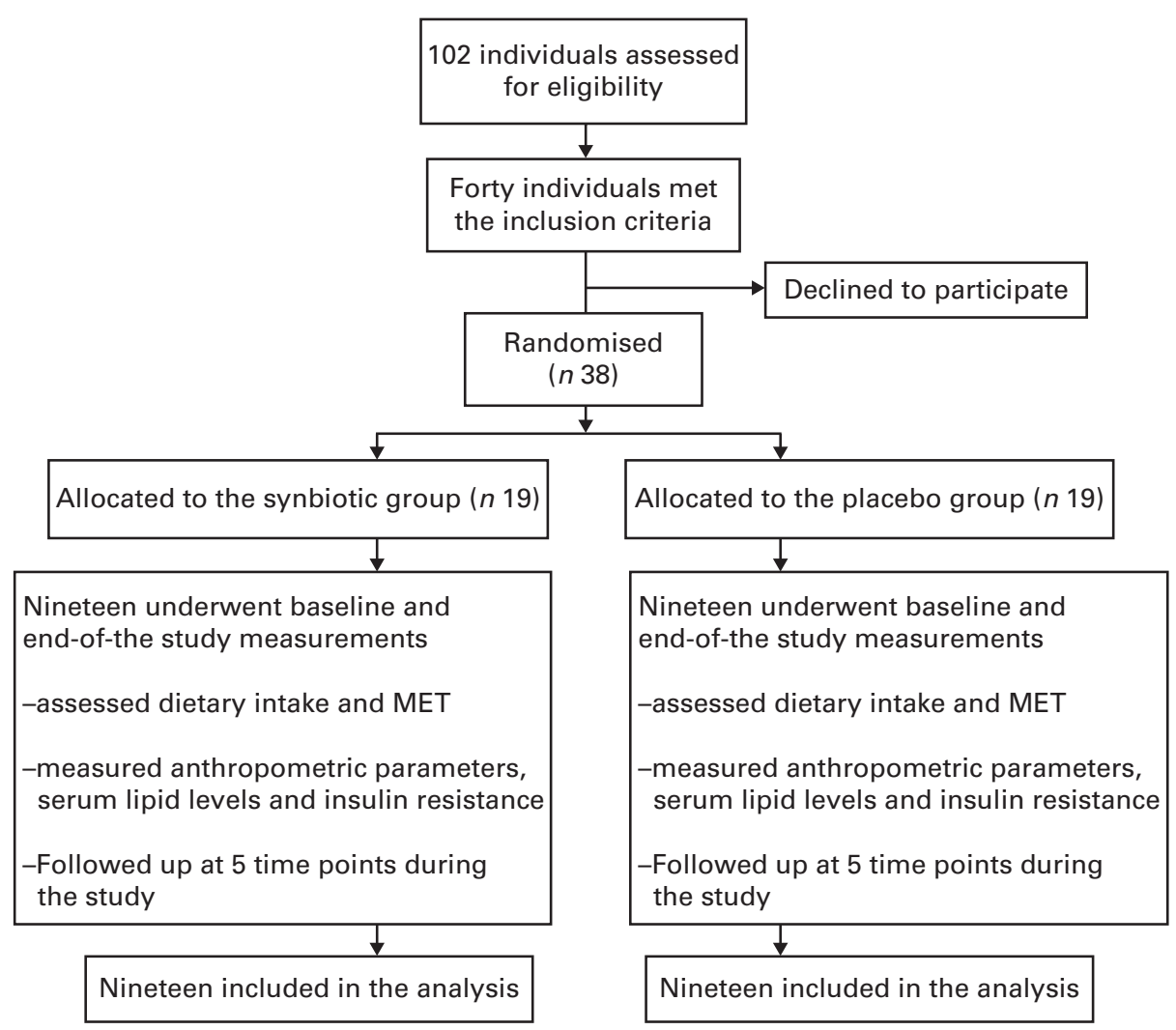

Fig. 1. Flow chart depicting the study design.

\section{Measurement of anthropometric parameters and blood pressure}

Anthropometric measurements of height and weight, as well as waist and hip circumferences, were made at baseline. The measurements were repeated at weeks 7, 14, 21 and 28. Height was measured to the nearest $0.5 \mathrm{~cm}$; weight was measured in light clothing and barefoot to the nearest $0 \cdot 1 \mathrm{~kg}$. Waist circumference was measured to the nearest $0 \cdot 1 \mathrm{~cm}$ using a flexible tape at the mid-point between the lowest rib and the iliac crest. Hip circumference was measured around the widest portion of the buttocks, with the tape parallel to the floor. The waist:hip ratio was calculated according to WHO recommendations ${ }^{(22)}$. BMI was also calculated by dividing each participant's weight in kg by his or her height in $\mathrm{m}^{2}$. All the measurements were taken by the same person to decrease the error rate. Each participant's blood pressure was measured by a trained nurse on two occasions, before and after sitting for a $15 \mathrm{~min}$ period, using a manual blood pressure cuff; the mean blood pressure reading was used for the present analysis ${ }^{(23)}$

\section{Assessment of dietary intake}

Dietary intake was assessed at five time points during the course of the study using food records. At each time point, the participants were instructed to record their daily food and beverage intake for $3 \mathrm{~d}$, including a weekend day. Food scales and models were also used to enhance portion size accuracy. For standardisation purposes, portion sizes were converted from household measurements to grams, and every food and beverage item was subsequently coded according to the protocol and analysed for energy content and other nutrients using Nutritionist 4 (First DataBank), which was modified using the national composition food tables $^{(24)}$. Physical activity was also assessed using the metabolic equivalent of task (MET) questionnaire ${ }^{(25)}$ at weeks 0 , 7, 14, 21 and 28.

\section{Measurement of biochemical parameters}

Each participant underwent a biochemical test at baseline, during each follow-up visit and at the end of the study. After a $12 \mathrm{~h}$ fast, blood was drawn from the antecubital vein into evacuated tubes containing EDTA, and serum lipid profile, fasting blood sugar levels and insulin levels were determined. All the biochemical assessments were carried out in the same laboratory using standard laboratory methods. Serum TAG, HDL and total cholesterol (TC) levels were determined using a photometric assay (Reckon), while LDLcholesterol levels were determined using the following equation: $\quad \mathrm{LDL}=\mathrm{TC}-\mathrm{HDL}-0 \cdot 16(\mathrm{TAG})$. Hypertriacylglycerolaemia was defined as having fasting TAG levels $\geq 150 \mathrm{mg} / \mathrm{dl}$ $(\geq 1.69 \mathrm{mmol} / \mathrm{l})$ and hypercholesterolaemia as having fasting cholesterol levels $>200 \mathrm{mg} / \mathrm{dl}(>5.17 \mathrm{mmol} / \mathrm{l})$. Fasting HDL levels $<50 \mathrm{mg} / \mathrm{dl}(<1.29 \mathrm{mmol} / \mathrm{l})$ for women and $<40 \mathrm{mg} / \mathrm{dl}$ $(<1.03 \mathrm{mmol} / \mathrm{l})$ for men were considered to be low, and LDL levels $\geq 130 \mathrm{mg} / \mathrm{dl} \quad(\geq 3.36 \mathrm{mmol} / \mathrm{l})$ were considered 
to be high. Fasting glucose levels were measured using the GOD/POD method. Fasting insulin levels were measured using ELISA (Mercodia AB).

\section{HOMA-IR index}

The HOMA-IR index was used to determine the degree of insulin resistance using the following formula ${ }^{(26)}$

$$
\begin{aligned}
\text { HOMA }-\mathrm{IR}= & (\text { fasting blood glucose }(\mathrm{mmol} / \mathrm{l}) \\
& \times \text { fasting insulin }(\mu \mathrm{U} / \mathrm{ml})) / 22 \cdot 5 .
\end{aligned}
$$

\section{QUICKI index}

Insulin sensitivity was assessed using the quantitative insulin sensitivity check index (QUICKI) equation: QUICKI $=1 /(\log$ insulin $(\mu \mathrm{U} / \mathrm{ml})+\log$ glucose $(\mathrm{mg} / \mathrm{dl}))^{(27)}$. A low QUICKI index indicates low insulin sensitivity, while a high QUICKI index indicates high insulin sensitivity.

The conversion factor for glucose is $\mathrm{mg} / \mathrm{dl}=\mathrm{mmol} / \mathrm{l} \times 18$ and for insulin is $\mu \mathrm{U} / \mathrm{ml}=\mathrm{pmol} / 1 / 6.9$

\section{Statistical analyses}

Data were analysed using the STATA software (version 11, StataCorp). For all analyses, a $P$ value $<0.05$ was considered statistically significant. Continuous and categorical data are reported as means and standard deviations and frequency (\%), respectively. Demographic variables were analysed using $\chi^{2}$ or $t$ test, as appropriate. Pearson's correlation coefficient was calculated to assess the relationship between some outcomes. Means and 95\% CI for changes in outcome variables from baseline were compared at 7, 14, 21 and 28 weeks using ANCOVA models at each time point. All the ANCOVA models were adjusted for sex, age, baseline value of each outcome and mean change in BMI, waist:hip ratio,
MET and energy. Means and 95\% CI for HOMA-IR and QUICKI values were plotted $v$. time from baseline to the end of the treatment.

The sample size was obtained of eighteen subjects per group to discover a difference of $10 \%$ on the mean values of HOMA-IR ${ }^{(28)}$ between the G1 and G2 groups after 28 weeks of treatment, to a probability of error $5 \%$ and to a test power $80 \%$.

\section{Results}

\section{Characteristics of the participants}

The inclusion criteria of the study were met by thirty-eight participants, and they were included in the analysis. No participant was lost to follow-up in the present study. A flow chart depicting the study design is shown in Fig. 1. The baseline clinical and demographic characteristics of both groups are summarised in Table 1. Among the participants, 8\% were smokers and $60.53 \%$ were female. The baseline anthropometric characteristics of both groups were similar (Table 1).

\section{Findings recorded in the two groups of participants before and after treatment}

All the participants completed the trial. From baseline to the end of the study, both groups exhibited similar reductions in many of the study parameters. The in-between group changes were as follows: BMI - 32.13 (sD 2.7) to $30 \cdot 91(\mathrm{sD} 2 \cdot 5) \mathrm{kg} / \mathrm{m}^{2}$ in the G1 group $v .31 .51$ (SD 1.47$)$ to $30.37(\mathrm{SD} 2 \cdot 2) \mathrm{kg} / \mathrm{m}^{2}$ in the G2 group; $P=0.656$; waist girth -103.42 (SD 7.5) to 101.42 (SD 6.5$) \mathrm{cm}$ in the G1 group $v .102 .37$ (SD 6.4) to 99.79 (SD 5.7$) \mathrm{cm}$ in the G2 group; $P=0.516$; energy intake -9785.5 (SD 2351) to $8104 \cdot 28(\mathrm{SD} 1465 \cdot 6) \mathrm{kJ} / \mathrm{d}$ in the G1 group $v$. $9294.63(\mathrm{SD} 1764.8)$ to $7933.95(\mathrm{sD} 1271.3) \mathrm{kJ} / \mathrm{d}$ in the G2 group; $P=0.284 ;$ MET -32.25 (SD 4.25 ) to 33.93

Table 1. Baseline characteristics of the study participants

\begin{tabular}{|c|c|c|c|c|c|c|c|}
\hline & \multicolumn{2}{|c|}{$\begin{array}{l}\text { Synbiotic group } \\
(\text { (n 19) }\end{array}$} & \multicolumn{2}{|c|}{$\begin{array}{l}\text { Placebo group } \\
\quad(n 19)\end{array}$} & \multicolumn{2}{|c|}{ Total $(n 38)$} & \multirow[b]{2}{*}{$P$} \\
\hline & Mean & SD & Mean & SD & Mean & SD & \\
\hline Age (years) & 47.52 & $9 \cdot 1$ & 46.05 & $10 \cdot 1$ & 46.79 & 9.5 & 0.638 \\
\hline Waist girth $(\mathrm{cm})$ & 103.42 & $7 \cdot 51$ & $102 \cdot 37$ & 6.40 & $102 \cdot 89$ & 6.90 & 0.645 \\
\hline BMI $\left(\mathrm{kg} / \mathrm{m}^{2}\right)$ & $32 \cdot 13$ & $2 \cdot 7$ & 31.51 & $2 \cdot 3$ & 31.82 & 2.5 & 0.458 \\
\hline FBS (mmol/l) & 5.62 & $1 \cdot 12$ & $5 \cdot 77$ & 1.47 & 5.69 & 1.29 & 0.731 \\
\hline \multicolumn{8}{|c|}{ Blood pressure $(\mathrm{mmHg})$} \\
\hline Systolic & 124.53 & 10.93 & $127 \cdot 97$ & 18.43 & $126 \cdot 25$ & 15.05 & 0.488 \\
\hline Diastolic & 83.87 & 8.95 & 88.08 & $10 \cdot 21$ & 85.97 & $9 \cdot 71$ & 0.185 \\
\hline $\mathrm{TAG}(\mathrm{mmol} / \mathrm{l})$ & 2.58 & 0.6 & 2.66 & 1.04 & 2.62 & 0.84 & 0.769 \\
\hline $\mathrm{HDL}(\mathrm{mmol} / \mathrm{l})$ & $1 \cdot 15$ & 0.14 & 1.12 & 0.13 & 1.14 & 0.13 & 0.502 \\
\hline LDL (mmol/l) & 1.53 & 0.86 & 1.94 & $1 \cdot 13$ & 1.73 & 1.01 & 0.220 \\
\hline $\mathrm{TC}(\mathrm{mmol} / \mathrm{l})$ & 3.84 & 0.79 & 4.28 & 0.96 & 4.06 & 0.89 & 0.130 \\
\hline Insulin (pmol/l) & 71.39 & 33.34 & $75 \cdot 63$ & $24 \cdot 31$ & 73.48 & $29 \cdot 17$ & 0.661 \\
\hline HOMA-IR & 2.59 & 1.5 & $2 \cdot 81$ & $1 \cdot 1$ & 2.70 & 1.3 & 0.615 \\
\hline QUICKI & 0.34 & 0.03 & 0.33 & 0.02 & 0.34 & 0.02 & 0.311 \\
\hline MET/h per d & $32 \cdot 25$ & 4.2 & 31.47 & $4 \cdot 1$ & 31.86 & $4 \cdot 1$ & 0.572 \\
\hline Energy (kJ) & 9785.53 & 2350.9 & 9294.75 & $1764 \cdot 8$ & 9539.9 & $2065 \cdot 2$ & 0.471 \\
\hline
\end{tabular}
(Mean values and standard deviations)

FBS, fasting blood sugar; TC, total cholesterol; HOMA-IR, homeostasis model assessment - insulin resistance; QUICKI, quantitative insulin sensitivity check index; MET, metabolic equivalent of task. 
(SD 4.15) MET/h per $\mathrm{d}$ in the G1 group $v .31 .47$ (SD 4.13) to 33.06 (SD 4.32) MET/h per $\mathrm{d}$ in the $\mathrm{G} 2 ; P=0.524$ group.

On comparing the baseline and end-of-the-study results, a significant improvement in several metabolic parameters was observed within each of the groups and also between them. Although fasting blood sugar and insulin levels decreased significantly in both groups, this decrease was greater in the G1 group than in the G2 group, as follows: fasting blood sugar $-5.62(\mathrm{sD} 1.12)$ to $5 \cdot 16(\mathrm{sD} 0 \cdot 22) \mathrm{mmol} / \mathrm{l}$ in the G1 group $v$. 5.77 (SD 1.47 ) to 5.59 (SD 1.46$) \mathrm{mmol} / \mathrm{l}$ in the G2 group; $P<0.001$ (Table 2), and insulin -71.39 (sD 33.34) to 57.92 (SD 29.17) $\mathrm{pmol} / 1$ in the G1 group $v .75 .63$ (SD 24.31) to 68.82 (SD 22.92 ) $\mathrm{pmol} / 1$ in the $\mathrm{G} 2$ group; $P=0.014$. Insulin resistance also improved; the HOMA-IR index decreased from 2.59 (SD 1.5) to 1.94 (SD 1.1) in the G1 group and from $2 \cdot 8$ (SD 1.1) to 2.42 (SD 0.9) in the G2 group ( $P=0.046)$, while the QUICKI index increased from $0.342(\mathrm{SD} 0.027)$ to 0.357 (SD 0.028$)$ in the G1 group $v .0 .333$ (SD 0.022) to 0.339 (SD 0.024) $(P<0.001)$ in the G2 group (Fig. 2). A mean reduction of $11 \%$ in HOMA-IR ( $25 \%$ reduction in the G1 group and 14\% in the G2 group) was achieved, meeting our aim, which was set at $10 \%$.

On comparing the two groups, significant changes were also observed in serum lipid parameters. In both the G1 and G2 groups, particularly, there was a significant decrease in TAG levels $(2.58(\mathrm{sD} 0.6)$ to $1.78(\mathrm{sD} 0.54) \mathrm{mmol} / \mathrm{l}$ in the $\mathrm{G} 1$ group v. $2.66(\mathrm{SD} 1.04)$ to $2.54(\mathrm{SD} 1.09) \mathrm{mmol} / \mathrm{l}$ in the $\mathrm{G} 2$

Table 2. Mean values of some outcome variables at 7, 14, 21 and 28 weeks after baseline

(Mean values and standard deviations)

\begin{tabular}{|c|c|c|c|c|c|}
\hline & \multicolumn{2}{|c|}{$\begin{array}{l}\text { Synbiotic group } \\
(n 19)\end{array}$} & \multicolumn{2}{|c|}{$\begin{array}{l}\text { Placebo group } \\
\qquad(n 19)\end{array}$} & \multirow[b]{2}{*}{$P^{*}$} \\
\hline & Mean & SD & Mean & SD & \\
\hline \multicolumn{6}{|c|}{ FBS (mmol/l) } \\
\hline Week 7 & $5 \cdot 4$ & 1.04 & $5 \cdot 66$ & 1.48 & 0.048 \\
\hline Week 14 & $5 \cdot 35$ & 1.04 & 5.69 & 1.47 & 0.001 \\
\hline Week 21 & $5 \cdot 27$ & 1.08 & $5 \cdot 76$ & 1.47 & $<0.001$ \\
\hline Week 28 & $5 \cdot 16$ & 0.22 & 5.59 & 1.46 & $<0.001$ \\
\hline \multicolumn{6}{|c|}{ TAG $(\mathrm{mmol} / \mathrm{l})$} \\
\hline Week 7 & $2 \cdot 17$ & 0.74 & $2 \cdot 50$ & 1.01 & 0.008 \\
\hline Week 14 & 2.05 & 0.67 & $2 \cdot 40$ & $1 \cdot 16$ & 0.007 \\
\hline Week 21 & 1.90 & 0.60 & $2 \cdot 60$ & $1 \cdot 14$ & $<0.001$ \\
\hline Week 28 & 1.78 & 0.54 & 2.54 & 1.09 & $<0.001$ \\
\hline \multicolumn{6}{|l|}{$\mathrm{HDL}(\mathrm{mmol} / \mathrm{l})$} \\
\hline Week 7 & $1 \cdot 24$ & 0.12 & 1.09 & $0 \cdot 16$ & 0.003 \\
\hline Week 14 & $1 \cdot 28$ & 0.11 & $1 \cdot 10$ & 0.15 & $<0.001$ \\
\hline Week 21 & $1 \cdot 31$ & $0 \cdot 12$ & $1 \cdot 13$ & 0.19 & 0.004 \\
\hline Week 28 & $1 \cdot 35$ & 0.10 & $1 \cdot 12$ & 0.16 & $<0.001$ \\
\hline \multicolumn{6}{|l|}{$\mathrm{TC}(\mathrm{mmol} / \mathrm{l})$} \\
\hline Week 7 & 3.63 & 0.74 & $4 \cdot 20$ & 1.01 & 0.143 \\
\hline Week 14 & 3.60 & 0.82 & $4 \cdot 13$ & 1.03 & 0.884 \\
\hline Week 21 & 3.61 & 0.94 & $4 \cdot 16$ & $1 \cdot 18$ & 0.904 \\
\hline Week 28 & $3 \cdot 26$ & 0.73 & 3.92 & 0.93 & 0.010 \\
\hline \multicolumn{6}{|l|}{$\mathrm{LDL}(\mathrm{mmol} / \mathrm{l})$} \\
\hline Week 7 & 1.40 & 0.77 & 1.98 & $1 \cdot 14$ & 0.092 \\
\hline Week 14 & 1.41 & 0.86 & 1.93 & $1 \cdot 17$ & 0.256 \\
\hline Week 21 & 1.49 & 0.96 & 1.85 & $1 \cdot 27$ & 0.714 \\
\hline Week 28 & $1 \cdot 14$ & 0.78 & 1.64 & 1.05 & 0.347 \\
\hline
\end{tabular}

FBS, fasting blood sugar; TC, total cholesterol.

* Based on ANCOVA model regressing change from baseline on the treatment group, baseline value of the outcome, sex, age, and mean change in BMI, waist, MET and energy. group; $P<0.001$ ) and TC levels (3.84 (SD 0.79) to 3.26 (SD 0.73$) \mathrm{mmol} / \mathrm{l}$ in the G1 group $v .4 .28$ (SD 0.96) to 3.92 (sD 0.93 ) $\mathrm{mmol} / \mathrm{l}$ in the G2 group; $P=0.013$ ). Serum HDL levels differed significantly between the G1 and G2 groups $(0 \cdot 2$; from $1.15(\mathrm{sD} 0 \cdot 14)$ to $1.35(\mathrm{sD} 0 \cdot 1) \mathrm{mmol} / \mathrm{l}$ in the $\mathrm{G} 1$ group $v$. $<0.01$; from $1.12(\mathrm{SD} 0.13$ ) to $1.12(\mathrm{SD} 0 \cdot 16) \mathrm{mmol} / \mathrm{l}$ in the G2 group; $P<0 \cdot 001$ ). This difference was due to the significant increase in HDL levels in the G1 group, while its levels remained unchanged in the G2 group. There were no significant changes in serum LDL levels within and between the groups before and after adjustments (Table 2). All the observed differences in the study parameters, except for TC, were significant between the two groups starting at week 7 of the treatment. TC levels decreased significantly after week 21 .

None of the participants who completed the trial had any serious adverse events, indicating tolerance to the treatment. In the G1 group, one participant complained of moderate headache. In the G2 group, one participant reported abdominal pain. Neither complaint resulted in participant dropout from the study or reoccurred.

\section{Discussion}

To our knowledge, this is the first randomised, double-blind, placebo-controlled clinical study to demonstrate that a synbiotic supplement can increase the efficacy of conventional lifestyle modification in the treatment of the metabolic syndrome. Synbiotic supplementation for 28 weeks led to significant improvements in insulin resistance indices and TAG, TC and HDL levels, while LDL levels remained unchanged. This efficacy of treatment can at least partially be attributed to the effects of the synbiotic on gut flora modification, further causing alterations in carbohydrate absorption, improvement of insulin resistance or modulation of inflammatory signalling pathways $^{(29,30)}$.

The effects of synbiotic therapy on insulin resistance, one of the defining features of the metabolic syndrome, have only been investigated in a small number of studies. In the present study, we were able to demonstrate the significant positive effects of synbiotic supplementation on insulin resistance. When comparing healthy individuals with individuals having diabetes mellitus or insulin resistance, studies have found LPS to be present at higher levels in the blood of the latter group ${ }^{(31)}$. Circulating LPS levels have also been found to be correlated with insulin and glucose levels and HOMA$\mathrm{IR}^{(7)}$. Interventional studies suggest that such increases in LPS levels may play a direct role in the progression of insulin resistance, as a bolus injection of LPS into healthy subjects has been shown to cause a $35 \%$ reduction in frequently sampled intravenous glucose tolerance, used for measuring the insulin sensitivity index ${ }^{(32)}$. Possible mechanisms by which synbiotic supplementation can improve insulin resistance are as follows: through the modification of gut flora and the reduction of endotoxin levels and also through the reduction of the production and absorption of intestinal toxins and elevation of faecal $\mathrm{pH}^{(33)}$. All these mechanisms can suppress and modify small intestinal bacterial overgrowth, including the reduction of the abundance of Gram-negative bacteria and 
(a)

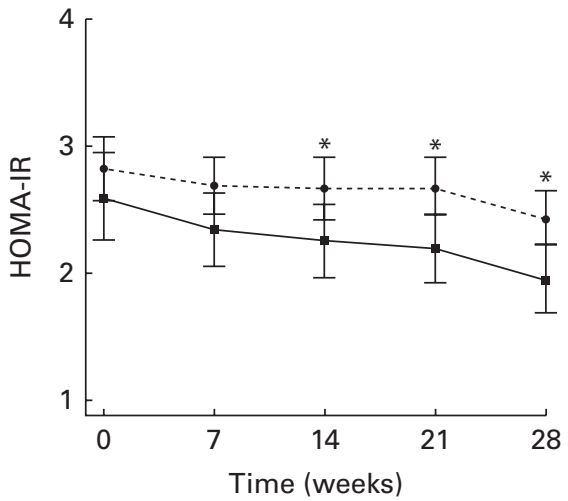

(b)

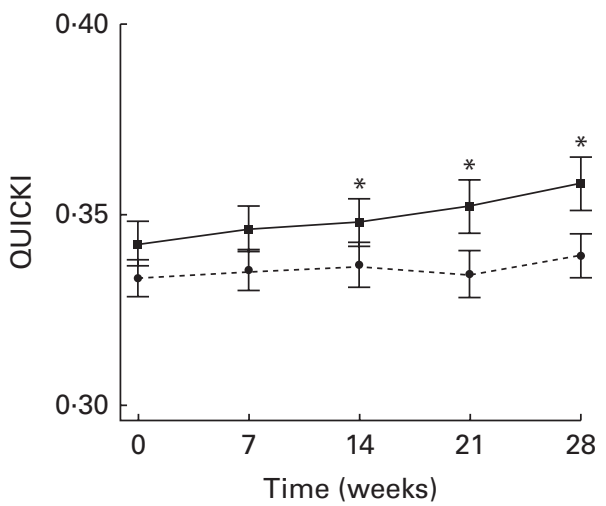

Fig. 2. Mean values obtained for the (a) homeostasis model assessment - insulin resistance (HOMA-IR) index and (b) quantitative insulin sensitivity check index (QUICKI) during treatment and follow-up. The number of participants at each visit and within each treatment group was 19 . Error bars indicate $95 \%$ CI. * $P<0.05$.

their by-products such as LPS while at the same time causing the reduction of proinflammatory cytokine production, leading to a decrease in fasting insulin levels and consequently insulin resistance ${ }^{(16,34)}$.

The results of the present study indicate a significant reduction in fasting blood sugar and insulin levels, as also reflected by improvements in HOMA-IR and QUICKI indices. Malaguarnera et $a l^{(18)}$ also observed a significant reduction in the HOMA-IR index, but no significant change in serum insulin levels. This could partially be explained by the fact that Malaguarnera et $a l^{(18)}$ used one strain of probiotics B. longum - along with fructo-oligosaccharide, but in the present study several strains were used together, possibly contributing to the significant differences observed in insulin levels. Yet other studies using other strains of probiotics have also found significant reductions in insulin resistance. For instance, Yadav et al. ${ }^{(35)}$ observed this change in high-fructose dietfed mice that were supplemented with dahi containing L. acidophilus. The results of the present study are consistent with those of previous studies showing different strains of bacteria to reduce insulin resistance in animals and human subjects with other metabolic disorders ${ }^{(35,36)}$.

Although there were significant changes in HDL, TC and TAG levels in both groups in the present study, the mean changes were greater in the G1 group than in the G2 group. In a randomised, placebo-controlled study involving twentynine women, using synbiotic-containing yogurt, Kiessling et $a l^{(37)}$ also found a significant increase in HDL-cholesterol levels. The increase in serum HDL levels in the G1 group can be attributed to the reduction in serum TAG levels, as a reduction in TAG levels can affect HDL levels. The mechanism responsible for this change has not been elucidated yet; however, possible mechanisms have been suggested. One of these mechanisms is that TAG-rich lipoprotein particles such as VLDL and intermediate-density lipoprotein can exchange TAG for cholesteryl esters from HDL particles, in a process catalysed by cholesteryl ester transfer proteins. This process occurs at a higher rate in hypertriacylglycerolaemic states and HDL particles become TAG-rich, cholesterol-poor HDL particles, which are better substrates for hepatic lipases. These enzymes, through lipolysis of TAG, transform TAG-rich, cholesterol-poor HDL particles into small, dense HDL particles, which are catabolised more rapidly than their larger counterparts, consequently leading to reduced serum HDL levels ${ }^{(38)}$.

Although other studies had also found a significant decrease in LDL levels ${ }^{(39,18)}$, in the present study, LDL levels were not found to change significantly. This may be because the baseline LDL levels of the participants of the present study were within the normal range, whereas those of the participants of other studies were above the normal range ${ }^{(18)}$. Therefore, it is possible that normal LDL levels are not affected by probiotics.

Some of the important strengths of the present study include its relatively long duration, the evaluation of insulin resistance indices, its randomised design, and the inclusion of subjects newly diagnosed with the metabolic syndrome who had not yet received any treatment for their conditions. Also, discrepancies in the composition of the two groups were minimised because the patient population was well defined by the inclusion and exclusion criteria.

The present study also has some limitations. Faecal bacterial loads were not measured before and after synbiotic treatment. Another limitation is that the study participants were not followed up further to test the sustainability of the results obtained after the termination of synbiotic treatment. Future randomised, placebo-controlled studies should also include a washout period.

In conclusion, this randomised, double-blind, placebocontrolled study has found some evidence that synbiotic supplementation augments the effects of lifestyle modification in the treatments of the metabolic syndrome at least partially through the attenuation of insulin resistance and serum lipid levels.

\section{Acknowledgements}

The present study was supported by a grant from the NNFTRI and the DDRI. The authors are grateful to the Protexin Company, UK, for gifting the synbiotic supplements. They are indebted to the Haraz clinic at the 17-Shahrivar Hospital in Amol for providing the equipment used in the study. 
They also thank Mr Mostafa Fallah, Mrs Farzaneh Zamani and Ms Mansoureh Maadi for their assistance.

The authors' contributions are as follows: T. E., A. H. and H. P. designed the study protocol and prepared the initial draft of the manuscript; T. E., A. H., M. S. and H. P. contributed to the data analysis and interpretation of results; F. Z. and R. M. ethically and financially supported the study; T. E., F. Z., A. H., M. S., S. E., R. M. and H. P. contributed to the writing and editing of the manuscript. All authors had access to all data and had final responsibility for the manuscript content, while H. P. and A. H. had final responsibility for the decision to submit the manuscript for publication.

None of the authors has any conflicts of interest to declare.

\section{References}

1. Grundy SM (2004) Obesity, metabolic syndrome, and cardiovascular disease. J Clin Endocrinol Metab 89, 2595-2600.

2. Musso G, Gambino R \& Cassader M (2010) Gut microbiota as a regulator of energy homeostasis and ectopic fat deposition: mechanisms and implications for metabolic disorders. Curr Opin Lipidol 21, 76-83.

3. Tilg H (2010) Obesity, metabolic syndrome, and microbiota: multiple interactions. J Clin Gastroenterol 44, S16-S18.

4. Turnbaugh PJ (2009) A core gut microbiome in obese and lean twins. Nature 457, 480-484.

5. Ley RE (2010) Obesity and the human microbiome. Curr Opin Gastroenterol 26, 5-11.

6. Al-Attas OS, Al-Daghri NM, Al-Rubeaan K, et al. (2009) Changes in endotoxin levels in T2DM subjects on antidiabetic therapies. Cardiovasc Diabetol 8, 20.

7. Ghanim H (2009) Increase in plasma endotoxin concentrations and the expression of Toll-like receptors and suppressor of cytokine signaling-3 in mononuclear cells after a high-fat, high-carbohydrate meal: implications for insulin resistance. Diabetes Care 32, 2281-2287.

8. Wendel M, Paul R \& Heller AR (2007) Lipoproteins in inflammation and sepsis. II. Clinical aspects. Intens Care Med 33, $25-35$.

9. Vasiljevic T \& Shah NP (2008) Probiotics - from Metchnikoff to bioactives. Int Dairy J 18, 714-728.

10. Cani PD (2006) Improvement of glucose tolerance and hepatic insulin sensitivity by oligofructose requires a functional glucagon-like peptide 1 receptor. Diabetes 55, 1484-1490.

11. Andreasen AS (2010) Effects of Lactobacillus acidophilus NCFM on insulin sensitivity and the systemic inflammatory response in human subjects. Br J Nutr 104, 1831-1838.

12. Bogsan CSB, Florence ACR, Perina N, et al. (2011) Probiotics intake and metabolic syndrome: a proposal. Trends Food Sci Technol 22, 457-464.

13. Frece J, Kos B, Svetec IK, et al. (2009) Synbiotic effect of Lactobacillus helveticus $\mathrm{M} 92$ and prebiotics on the intestinal microflora and immune system of mice. J Dairy Res 76, 98-104

14. Worthley DL, Le Leu RK, Whitehall VL, et al. (2009) A human, double-blind, placebo-controlled, crossover trial of prebiotic, probiotic, and synbiotic supplementation: effects on luminal, inflammatory, epigenetic, and epithelial biomarkers of colorectal cancer. Am J Clin Nutr 90, 578-586.

15. National Cholesterol Education Program (NCEP) Expert Panel on Detection E, and Treatment of High Blood Cholesterol in Adults (Adult Treatment Panel III) (2002) Third Report of the National Cholesterol Education Program (NCEP) Expert Panel on Detection, Evaluation, and
Treatment of High Blood Cholesterol in Adults (Adult Treatment Panel III) final report. Circulation 106, 3143-3421.

16. Li Z, Yang S, Lin H, et al. (2003) Probiotics and antibodies to TNF inhibit inflammatory activity and improve nonalcoholic fatty liver disease. Hepatology 37, 343-350.

17. Chen JJ, Wang R, Li X-F, et al. (2011) Bifidobacterium longum supplementation improved high-fat-fed-induced metabolic syndrome and promoted intestinal Reg I gene expression. Exp Biol Med 236, 823-831.

18. Malaguarnera M, Vacante M, Antic T, et al. (2012) Bifidobacterium longum with fructo-oligosaccharides in patients with non alcoholic steatohepatitis. Dig Dis Sci 57, 545-553.

19. The National Heart Lung and Blood Institute Expert Panel on the Identification, Evaluation, and Treatment of Overweight and Obesity in Adults (1998) Executive summary of the clinical guidelines on the identification, evaluation, and treatment of overweight and obesity in adults. J Am Diet Assoc 98, 1178-1191.

20. National Institutes of Health, National Heart, Lung, and Blood Institute, NHLBI Obesity Education Initiative, North American Association for the Study of Obesity (2000) The Practical Guide: Identification, Evaluation, and Treatment of Overweight and Obesity in Adults. Bethesda, MD: NIH.

21. Institute of Medicine of the National Academies (2002) Dietary Reference Intakes for Energy, Carbohydrate, Fiber, Fat, Fatty Acids, Cholesterol, Protein, and Amino Acids. Washington, DC: The National Academies Press.

22. World Health Organization (2012) STEPwise Approach to Surveillance (STEPS). Geneva: World Health Organization.

23. Hardy R, Kuh D, Langenberg C, et al. (2003) Birthweight, childhood social class, and change in adult blood pressure in the 1946 British birth cohort. Lancet 362, 1178-1183.

24. Ghaffarpour M, Houshiar-Rad A \& Kianfar H (1999) The Manual for Household Measures, Cooking Yields Factors and Edible Portion of Foods. Tehran: Nashre Olume Keshavarzy.

25. Ainsworth BE, Haskell WL, Whitt MC, et al. (2000) Compendium of physical activities: an update of activity codes and MET intensities. Med Sci Sports Exerc 32, 498-504.

26. Matthews D, Hosker J, Rudenski A, et al. (1985) Homeostasis model assessment: insulin resistance and $\beta$-cell function from fasting plasma glucose and insulin concentrations in man. Diabetologia 28, 412-419.

27. Hřebíček J, Janout V, Malinčíková J, et al. (2002) Detection of insulin resistance by simple quantitative insulin sensitivity check index QUICKI for epidemiological assessment and prevention. J Clin Endocrinol Metab 87, 144-147.

28. Aller R, De Luis D, Izaola O, et al. (2011) Effect of a probiotic on liver aminotransferases in nonalcoholic fatty liver disease patients: a double blind randomized clinical trial. Eur Rev Med Pharmacol Sci 15, 1090-1095.

29. O'Hara AM \& Shanahan F (2006) The gut flora as a forgotten organ. EMBO Rep 7, 688-693.

30. Neish AS (2009) Microbes in gastrointestinal health and disease. Gastroenterology 136, 65-80.

31. Pickup JC (2004) Inflammation and activated innate immunity in the pathogenesis of type 2 diabetes. Diabetes Care 27, 813-823.

32. Mehta NN, McGillicuddy FC, Anderson PD, et al. (2010) Experimental endotoxemia induces adipose inflammation and insulin resistance in humans. Diabetes 59, 172-181.

33. Compare D, Coccoli P, Rocco A, et al. (2012) Gut-liver axis: the impact of gut microbiota on non alcoholic fatty liver disease. Nutr Metab Cardiovasc Dis 22, 471-476. 
34. Peraldi P \& Spiegelman B (1998) TNF- $\alpha$ and insulin resistance: summary and future prospects. Mol Cell Biochem 182, 169-175.

35. Yadav H, Jain S \& Sinha PR (2007) Antidiabetic effect of probiotic dahi containing Lactobacillus acidophilus and Lactobacillus casei in high fructose fed rats. Nutrition 23, 62-68.

36. Ma X, Hua J \& Li Z (2008) Probiotics improve high fat dietinduced hepatic steatosis and insulin resistance by increasing hepatic NKT cells. J Hepatol 49, 821-830.

37. Kiessling G, Schneider J \& Jahreisl G (2002) Original Communication. Long-term consumption of fermented dairy products over 6 months increases HDL cholesterol. Eur J Clin Nutr 56, 843-849.

38. Khalatbari Soltani S, Jamaluddin R, Tabibi H, et al. (2012) Effects of flaxseed consumption on systemic inflammation and serum lipid profile in hemodialysis patients with lipid abnormalities. Hemodial Int 17, 275-281.

39. Schaafsma G, Meuling W, Van Dokkum W, et al. (1998) Effects of a milk product, fermented by Lactobacillus acidophilus and with fructo-oligosaccharides added, on blood lipids in male volunteers. Eur J Clin Nutr 52, 436-440. 